\title{
Constrained-Group-Delay-Optimized Multiple-Resonator-Based Harmonic Analysis
}

\author{
Miodrag D. KUŠLJEVIĆ* , Josif J. TOMIĆ, Predrag D. POLJAK
}

\begin{abstract}
Recently, a multiple-resonator structure was proposed as a robust and computationally efficient tool for harmonic analysis. Two trivial cases have been previously observed. The first case exhibits good out-of-band suppression and elimination of unwanted harmonics, but with a high latency. In the second case, a phase frequency response around the passband centre is zero-flat, that provides fast estimates. However, in this case, resonant peaks at the ends of the passband and high interharmonic gains cause large overshoots. In general, the basic algorithm performance requirements: selectivity and speed, are contradictory which makes it impossible to completely fulfil both of them. In this paper, the Constrained Linear Least-Squares (CLLS) optimization method is used to obtain a compromise solution. As a result, the resonant peaks in the passband are avoided and side lobes are mitigated, simultaneously minimizing the group delay in the middle of the passband. Performed simulations confirmed the effectiveness of the proposed algorithm.
\end{abstract}

Keywords: Constrained Linear Least-Squares (CLLS); frequency response; group delay; harmonic analysis; multiple-resonator; recursive algorithm

\section{INTRODUCTION}

It is widely accepted that, in practice, the voltage and current harmonics are time variant due to the dynamic nature of linear and nonlinear loads and equipment in power systems and the continuous changes in system configurtion. The analysis of the time-varying characteristics of harmonic distortion is an important research topic for protection and control of power systems [1]. Based on this fact, there has been an increasing interest in signal processing techniques for detecting and estimating harmonic components of time parameters. The challenge is designing a harmonic estimator with high convergence ratio, high acuracy, low computational burden and immunity to the presence of interharmonics: conditions that are not easy to simultaneously deal with. In general, the design difficulty of these systems lies on the trade-off between a fast-dynamic response and filtering performance. In order to obtain a fast-dynamic response, a wide bandwidth is required. On the other hand, to filter interference signals and noise, bandwidth should be reduced as much as possible. This yields a tight constraint, which can be hardly fulfilled.

A lot of various methods have been proposed to analyse the time-varying harmonics. Different discrete Fourier transform (DFT) based methods modified by pre-prcessing and postprocessing [2-7], demodulation based techniques [8], Kalman filtering [9-11], notch filters [12], recursiveleast-square (RLS) based [13-15] and least-mean-square (LMS) based algorithms [16], phase-locked loops [17-20], time-frequency representation [21], filter banks [22-25], discrete Taylor-Fourier transform (TFT) [26], Taylor-Kalman-Fourier (TKF) filter [27, 28], adapive cascaded filters [29], and other signal processing methods have been proposed recently in the literature to provide a more in-depth analysis of time varying harmonic distortion. The proposed techniques are more appropriate under dynamic conditions than the DFT which exhibits its limitations when applied to analyse non-stationary data. The DFT assumes the analysed signal is stationary and, hence, it is not suitable for computing time-varying harmonics. Good survey of some techniques is presented in [30].

One of the new approaches to the estimation of harmonics of dynamic signals based on recursive filters realized using multiple resonators (MR) was proposed in [31].
This algorithm, in addition to the fundamental and harmonics phasors, can also estimate their first derivatives (the first, second and so on, depending on multiplicity of resonators). Two signals are present at the output of the parallel resonator structure for each harmonic. The first one is the output of the cascade, and it shows good attenuation of noise and harmonics, but it has a large latency in relation to the input signal. In the case of the second signal, which is a sum of outputs of all resonators belonging to the corresponding cascade, a resulted filter has a zero-flat phase response around the operation frequency and thus it provides a fast estimate, but with large overshoot caused by resonant peaks at the ends of the passband.

In [32], by expanding the estimation structure described in [31], a compromised solution is offered. This is made possible by using a linear combination of the outputs of the corresponding resonators in the cascade, instead of the sum. Both frequency responses, amplitude and phase, in the bandwidth range were taken into account. The group delay of the transfer function in the centre of the bandwidth was controlled simultaneously with a limitation of the peaks at the ends of the bandwidth. This is obtained through the shifting of the reference time point along the filters data window directly influencing the latency. The minimum value of the group delay for given conditions is determined heuristically.

A different design technique is proposed in this paper. The block diagram of the MR-based estimator is the same as in [32], but the proposed design method is different. Unlike a design method proposed in [32] which used the heuristic procedure, constrains on the amplitude frequency response and/or the group delay are prescribed in advance and an optimized frequency response is obtained corresponding to the chosen minimisation function. Design problems can be defined in different way for different purposes. Thus, on the basis of the basic MR structure, it is possible to obtain a whole bank of filters corresponding to a dozen of both stationary and dynamic conditions.

\section{DESIGN METHOD}

Fig. 1 depicts a diagram of the triple-resonator-based estimation structure. A channel corresponding to the harmonic $m$ consists of the cascade of triple resonators with 
pole $z_{m}=\exp \left(j m \omega_{1}\right)$ at an angular frequency $\omega_{m}=2 \pi m f_{1} / f_{s}$ ( $f_{1}$ is the fundamental frequency, $f_{s}=1 / T$ is a sampling rate, $T$ is a sampling period). Complex gains $g_{m, k}, m=-M, \ldots$, $0, \ldots, M, k=0,1,2$, are assigned to the corresponding resonators in the cascade. All resonator cascades are embedded into the common feedback loop. Due to the infinite feedback loop gains at frequencies of the resonator poles, the transfer functions of each harmonic channel have unit values in own frequencies. This feature does not depend on other parameters of the system. Further, zero-flat gains around other harmonic frequencies are provided ensuring reinforcing of the required attenuations.

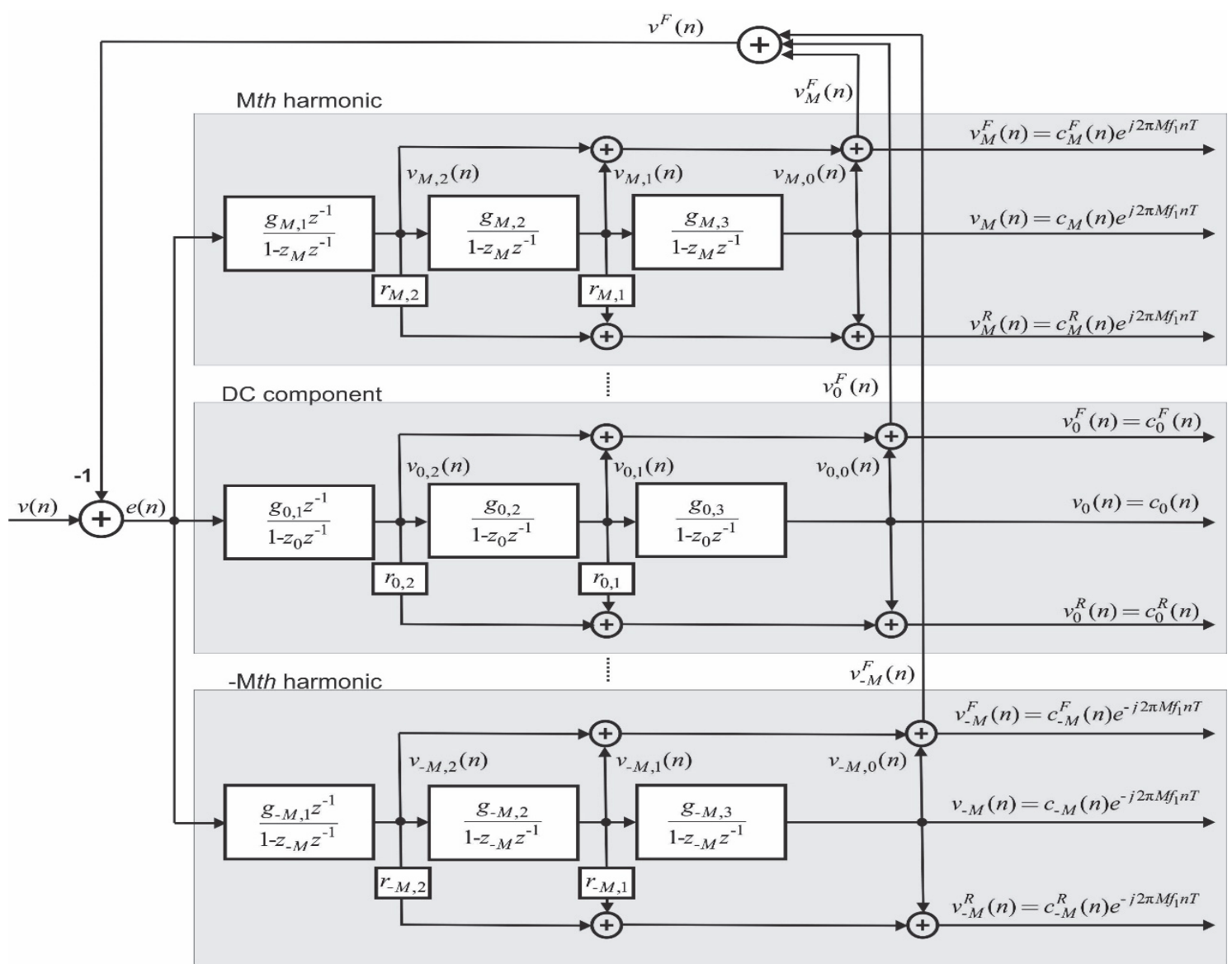

Figure 1 Block diagram of the third-order resonator estimation structure

The order of the overall closed system is $3(2 M+1)$. A maximum number of harmonics is $M$, that is equal to the number of resonator pairs, and depends on the fundamental component angular frequency the $\omega_{1}=2 \pi f_{1} / f_{s}$, because condition $M \omega_{1}<\pi$ has to be satisfied. In [31], a closed form solution for the calculations of the resonator gains $g_{m, 1}, g_{m, 2}$ and $g_{m, 3}$ for a dead-beat estimator aregiven:

$$
\begin{aligned}
g_{m, 3}^{\prime}= & \left.\frac{z^{3 N-2}}{P_{m}(z)}\right|_{z=z_{m}} \\
g_{m, 2}^{\prime}= & \frac{3 N z^{3 N-2}-\left.g_{m, 3}^{\prime} z \frac{\mathrm{d} P_{m}(z)}{d z}\right|_{z=z_{m}}-2 g_{m, 3}^{\prime}}{P_{m}(z)}\left|-2\left(g_{m, 2}^{\prime}+2 g_{m, 3}^{\prime}\right) z \frac{\mathrm{d} P_{m}(z)}{d z}\right|_{z=z_{m}}-g_{m, 2}^{\prime}-g_{m, 3}^{\prime} \\
g_{m, 1}^{\prime}= & \left.\frac{-g_{m, 3}^{\prime} z^{2} \frac{\mathrm{d}^{2} P_{m}(z)}{\mathrm{d} z^{2}}}{2 P_{m}(z)}\right|_{m}(3 N-1) z^{3 N-2}
\end{aligned}
$$

where:

$$
\begin{aligned}
& P_{m}(z)=\prod_{\substack{i=-M \\
i \neq m}}^{M}\left(z-z_{i}\right)^{3} \\
& \frac{\mathrm{d} P_{m}(z)}{\mathrm{d} z}=3 P_{m}(z) \sum_{\substack{i=-M \\
i \neq m}}^{M} \frac{1}{z-z_{i}}
\end{aligned}
$$$$
\frac{\mathrm{d}^{2} P_{m}(z)}{\mathrm{d} z^{2}}=3 P_{m}(z)\left[3\left(\sum_{\substack{i=-M \\ i \neq m}}^{M} \frac{1}{z-z_{i}}\right)^{2}-\sum_{\substack{i=-M \\ i \neq m}}^{M} \frac{1}{\left(z-z_{i}\right)^{2}}\right]
$$$$
g_{m, 1}^{\prime}=g_{m, 1}, g_{m, 2}^{\prime}=g_{m, 1} g_{m, 2} \text { and } g_{m, 3}^{\prime}=g_{m, 1} g_{m, 2} g_{m, 3}
$$$$
m=-M, \ldots, 0, \ldots, M \text {. }
$$

Finally, it is:

$g_{m, 1}=g_{m, 1}^{\prime}, g_{m, 2}=g_{m, 2}^{\prime} / g_{m, 1}^{\prime}, g_{m, 3}=g_{m, 3}^{\prime} / g_{m, 2}^{\prime}$ 

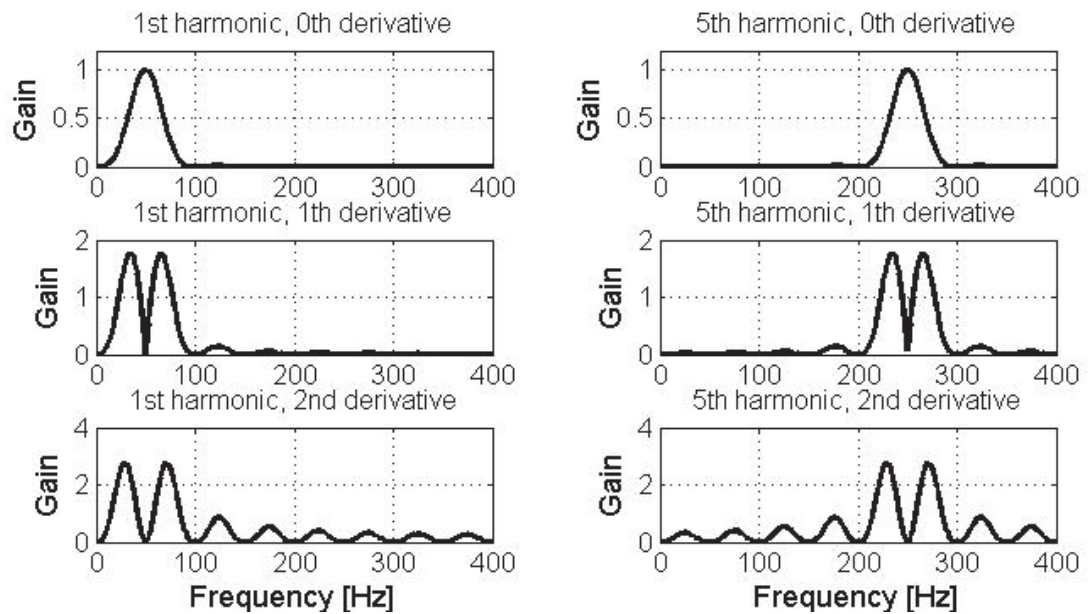

Figure 2 Frequency responses of the zero-, first and second-order derivatives (discrete FIR filters $T_{m, 0}(z), T_{m, 1}(z)$ and $T_{m, 2}(z)$ ) for $m=1$ and $m=5$, with $f_{1}=50 \mathrm{~Hz}$ and $f_{s}=$ $800 \mathrm{~Hz}$

It is supposed that a signal that will be analysed is defined by:

$$
v(n)=\sum_{m=-M}^{M} v_{m}(n)=\sum_{m=-M}^{M} c_{m}(n) \exp \left(j m \omega_{1} n T\right)
$$

The output of the $k$-th resonator (observed in the reverse order, starting from the end of the cascade and ending at its beginning) in the $m$-branch corresponding to the harmonic $m$ is

$v_{m, k}(n)=c_{m, k}(n) \exp \left(j m \omega_{1} n T\right)$

$m=-M, \ldots, 0, \ldots, M, k=0,1,2$.

It consists of the complex envelope $c_{m, k}(n)$ and constantly rotating vector $\exp \left(j m \omega_{1} n T\right)$. Notation $v_{m}(n)=v_{m, 0}(n)$ and $c_{m}(n)=c_{m, 0}(n)$ is also used.

The transfer functions corresponding to harmonic differentiator signals are as follows:

$T_{m, 0}(z)=\frac{V_{m, 0}(z)}{V(z)}=\frac{g_{m, 3}^{\prime} z^{2} P_{m}(z)}{z^{3(2 M+1)}}$

$T_{m, 1}(z)=\frac{V_{m, 1}(z)}{V(z)}=\frac{g_{m, 2}^{\prime} z\left(z-z_{m}\right) P_{m}(z)}{z^{3(2 M+1)}}$

$T_{m, 2}(z)=\frac{V_{m, 2}(z)}{V(z)}=\frac{g_{m, 1}^{\prime}\left(z-z_{m}\right)^{2} P_{m}(z)}{z^{3(2 M+1)}}$

Amplitude frequency responses of the transfer Eq. (6) to Eq. $(8)$ for the fundamental component $(m=1)$ and the fifth harmonic $(m=5)$ are given in Fig. 2 .

Two output signals belonging to the channel $m$ are noleontable: $\quad V_{m}(z)=V_{m, 0}(z)$ and $V_{m}^{F}(z)$. Signal $V_{m}^{F}(z)=V_{m, 0}(z)+V_{m, 1}(z)+V_{m, 2}(z)$ is inherently formed and embedded in a common feedback signal. Corresponding transfer functions are $T_{m, 0}(z)$ and
$T_{m}^{F}(z)=T_{m, 0}(z)+T_{m, 1}(z)+T_{m, 2}(z)$, respectively. Main good features and drawbacks of frequency responses of these two transfer functions are shown in [31]. The transfer function $T_{m, 0}(z)$ has a good amplitude frequency response providing the selectivity and good noise and harmonic/interhamonic attenuation. The drawback of this transfer function is its phase frequency response exhibiting a group delay of a half of the observation window. The transfer function $T_{m}^{F}(z)$ has a zero-flat phase frequency response around actual harmonic frequency which allows a fast (momentary) response. However, the huge disadvantages are large peaks at the ends the pass bands and high side lobes, Fig. 3.

The first two derivatives of the $T_{m}^{F}(z)$ in a pole $z_{m}$ equal zero. This property, together with a zero-flat phase frequency response around the pole $z_{m}$ (i.e. a zero-groupdelay in the pole $z_{m}$ ), is a reason for huge gain peaks at the ends of the passband width. The first attempt to reduce overshoots is to weak this requirement for the flatness. It can be shown that for one of the particular cases, a signal $V_{m}^{1 D}(z)=V_{m, 0}(z)+V_{m, 1}(z)$ (for $r_{m, 1}=1$ and $r_{m, 2}=1$ ), the first derivative of the corresponding transfer function $T_{m}^{1 D}(z)=T_{m, 0}(z)+T_{m, 1}(z)$ in zero pole $z_{m}$ equals zero, i.e. $\mathrm{d} T_{m}^{1 D}(z) /\left.\mathrm{d} z\right|_{z=z_{m}}=0$. In this case, the narrower both amplitude and phase flatness around the estimating harmonic frequency are provided. A gain of the amplitude response at the ends of the passband is smaller than for $T_{m}^{F}(z)$, but it is still too high and large resonant frequency peaks are still presented, Fig. 3 (solid line).

\subsection{Problem Statement}

In addition to inherent signals $V_{m, 0}(z)$ and $V_{m}^{F}(z)$, a new signal $V_{m}^{R}(z)=V_{m, 0}(z)+r_{m, 1} V_{m, 1}(z)+r_{m, 2} V_{m, 2}(z)$ is formed as a linear combination of the zero, first and second derivative estimates with coefficients $1, r_{m, 1}$ and $r_{m, 2}$, respectively. 

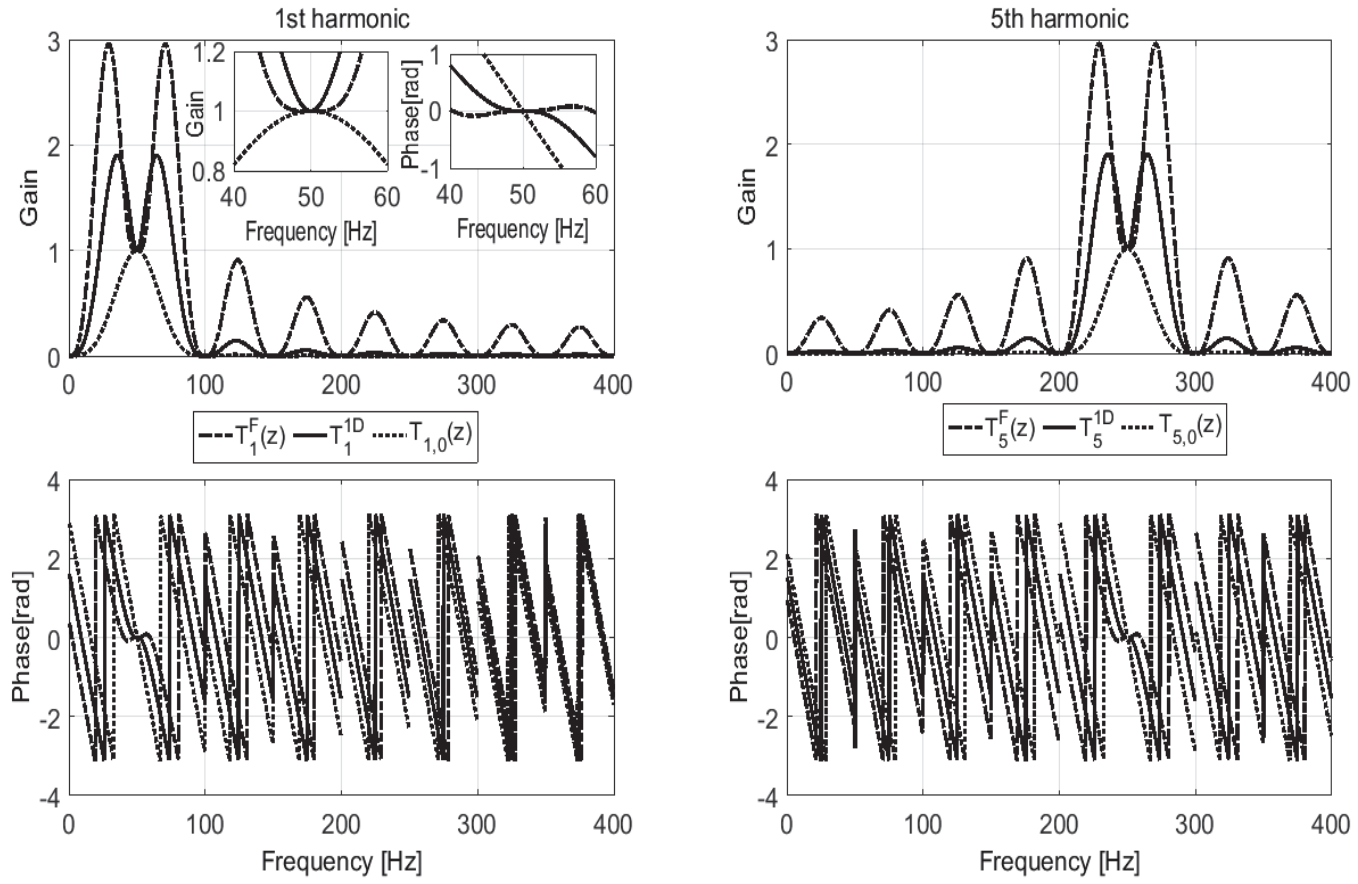

Figure 3 Frequency responses of the transfer functions $T_{m}^{F}(z), T_{m}^{1 D}(z)$ and $T_{m, 0}(z)$, corresponding to $m=1$ and $m=5$, with $f_{1}=50 \mathrm{~Hz}$ and $f_{s}=800 \mathrm{~Hz}$

The transfer function corresponding to the signal $V_{m}^{R}(z)$ is

$T_{m}^{R}(z)=\frac{V_{m}^{R}(z)}{V(z)}=T_{m, 0}(z)+r_{m, 1} T_{m, 1}(z)+r_{m, 2} T_{m, 2}(z)=$

$=\frac{\left[r_{m, 2} g_{m, 1}^{\prime}\left(z-z_{m}\right)^{2}+r_{m, 1} g_{m, 2}^{\prime} z\left(z-z_{m}\right)+g_{m, 3}^{\prime} z^{2}\right] P_{m}(z)}{z^{3(2 M+1)}}=$

$=\frac{R_{m}(z) P_{m}(z)}{z^{3(2 M+1)}}$

where:

$$
\begin{aligned}
R_{m}(z) & =r_{m, 2} g_{m, 1}^{\prime}\left(z-z_{m}\right)^{2}+r_{m, 1} g_{m, 2}^{\prime} z\left(z-z_{m}\right)+g_{m, 3}^{\prime} z^{2} \\
& =\left(r_{m, 2} g_{m, 1}^{\prime}+r_{m, 1} g_{m, 2}^{\prime}+g_{m, 3}^{\prime}\right) z^{2} \\
& -\left(2 r_{m, 2} g_{m, 1}^{\prime}+r_{m, 1} g_{m, 2}^{\prime}\right) z_{m} z+r_{m, 2} g_{m, 1}^{\prime} z_{m}^{2}
\end{aligned}
$$

Only coefficients of the residual (reshaping) polynomial $R_{m}(z)$, i.e. gain coefficients $\left\{r_{m, i}, i=1,2\right\}$, are subject to the optimization algorithm, since those of $P_{m}(z)$ are fixed for the selected frequency.

The first derivative of the transfer function $T_{m}^{R}(z)$ in the pole $z_{m}$ is:

$$
\begin{aligned}
& \frac{\mathrm{d} T_{m}^{R}\left(z_{m}\right)}{\mathrm{d} z}= \\
& =\left.\frac{\left\{\begin{array}{c}
r_{m, 1} g_{m, 2}^{\prime}\left(2 z-z_{m}\right) P_{m}(z)+g_{m, z^{\prime}}^{\prime} z^{2} \mathrm{~d} P_{m}(z) / \mathrm{d} z \\
-(6 M+1)) g_{m, 3}^{\prime} z P_{m}(z)
\end{array}\right.}{z^{3(2 M+1)}}\right|_{z=z_{m}}
\end{aligned}
$$

$\frac{\mathrm{d} T_{m}^{R}\left(z_{m}\right)}{\mathrm{d} z}=\boldsymbol{c}_{m} \boldsymbol{x}_{m}-d_{m}$

where:

$$
\begin{aligned}
& \boldsymbol{c}_{m}=\left[\begin{array}{ll}
g_{m, 2}^{\prime} P_{m}\left(z_{m}\right) / z_{m}^{(6 M+2)} & 0
\end{array}\right], \boldsymbol{x}_{m}=\left[\begin{array}{l}
r_{m, 1} \\
r_{m, 2}
\end{array}\right], \\
& d_{m}=\left[(6 M+1) P_{m}\left(z_{m}\right)-z_{m} \mathrm{~d} P_{m}\left(z_{m}\right) / \mathrm{d} z\right] g_{m, 3}^{\prime} / z_{m}^{(6 M+2)} .
\end{aligned}
$$

A further trade-off between the opposite requirements can be performed by minimization of $\left|\mathrm{d} T_{m}^{R}\left(z_{m}\right) / \mathrm{d} z\right|$ subject to limitation of gains in the parts of interest in the frequency response. It is requested that:

$$
\left|E_{m}\left(z_{i}\right)\right| \leq \delta_{m, i}, z_{i}=e^{j \omega_{i}}, i=1,2, \ldots, N
$$

where $E_{m}\left(z_{i}\right)=T_{m}^{R}\left(z_{i}\right)-H_{m}^{d}\left(z_{i}\right), H_{m}^{d}\left(z_{i}\right)$ are values of the prescribed (desired) transfer function $H_{m}(z)$ in points $z_{i}=e^{j \omega_{i}} \quad\left(H_{m}^{d}\left(z_{i}\right)=0\right)$, and $\left\{\delta_{m . i}, i=1,2, \cdots, N\right\}$ denotes a set of the prescribed bounds. $N$ is a number of selected frequencies of interest $(0 \leq \omega \leq \pi)$.

\subsection{Linearization of Constraints}

Eq. (12) are nonlinear. These constraints can be linearized using an appropriate approximation [33], allowing to solve the problem through a constrained linear least-squares (CLLS) optimization method [34]. In [33], an octagon has been used to approximate a circle. In this pa-

Eq. (10) has the following matrix form: 
per, an approximation of the circle is generalized to a polygon of any order providing an enough high approximation accuracy. It is easily proved to be valid:

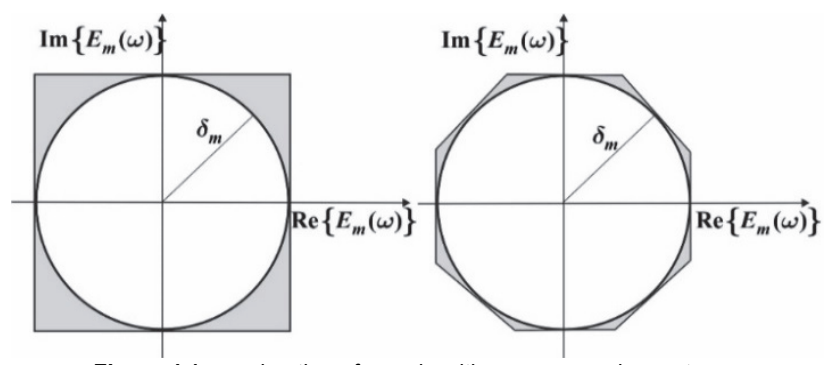

Figure 4 Approximation of a cycle with a square and an octagon

$\left|E_{m}\left(z_{i}\right)\right|=\operatorname{Re}\left\{E_{m}\left(z_{i}\right)\right\} \cos \alpha_{i}+\operatorname{Im}\left\{E_{m}\left(z_{i}\right)\right\} \sin \alpha_{i}$

where $\alpha_{i}=\arg \left(E_{m}\left(z_{i}\right)\right)$.

Since $\alpha_{i}$ is unknown, the following system of the linear inequalities can approximate nonlinear one (12):

$\operatorname{Re}\left\{T_{m}^{R}\left(z_{i}\right)\right\} \cos \alpha_{i j}+\operatorname{Im}\left\{T_{m}^{R}\left(z_{i}\right)\right\} \sin \alpha_{i j} \leq \delta_{m, i}$

where $i=1,2, \ldots, N$ and $j=1,2, \ldots, L$.

Usually angles $\alpha_{i j}=\alpha_{i 0}+(j-1) 2 \pi / L$ are taken, where $j=1,2, \ldots, L$. The number of linear constraints $L$ is determined by the requested tolerance. For a sufficient number of angles (which are convenient to be equally spaced) the linear system Eq. (14) approximates the Eq. (12) with the sufficient accuracy. Square and octagon approximations are shown in Fig. 4 . In this paper, $L=32$ is used.

The Eq. (14) can be written in a matrix form:

$$
\boldsymbol{A}_{m, i} \boldsymbol{x}_{m} \leq \boldsymbol{b}_{m, i}, i=1,2, \ldots, N
$$

$\boldsymbol{A}_{m, i}=\left[\begin{array}{c}\operatorname{Re}\left\{\boldsymbol{h}_{m, i}\right\} \cos \alpha_{i 1}+\operatorname{Im}\left\{\boldsymbol{h}_{m, i}\right\} \sin \alpha_{i 1} \\ \operatorname{Re}\left\{\boldsymbol{h}_{m, i}\right\} \cos \alpha_{i 2}+\operatorname{Im}\left\{\boldsymbol{h}_{m, i}\right\} \sin \alpha_{i 2} \\ \vdots \\ \operatorname{Re}\left\{\boldsymbol{h}_{m, i}\right\} \cos \alpha_{i L}+\operatorname{Im}\left\{\boldsymbol{h}_{m, i}\right\} \sin \alpha_{i L}\end{array}\right]$

$\boldsymbol{b}_{m, i}=\left[\begin{array}{c}\delta_{m, i}-\operatorname{Re}\left\{T_{m, 0}\left(z_{i}\right)\right\} \cos \alpha_{i 1}-\operatorname{Im}\left\{T_{m, 0}\left(z_{i}\right)\right\} \sin \alpha_{i 1} \\ \delta_{m, i}-\operatorname{Re}\left\{T_{m, 0}\left(z_{i}\right)\right\} \cos \alpha_{i 2}-\operatorname{Im}\left\{T_{m, 0}\left(z_{i}\right)\right\} \sin \alpha_{i 2} \\ \vdots \\ \delta_{m, i}-\operatorname{Re}\left\{T_{m, 0}\left(z_{i}\right)\right\} \cos \alpha_{i L}-\operatorname{Im}\left\{T_{m, 0}\left(z_{i}\right)\right\} \sin \alpha_{i L}\end{array}\right]$

$\boldsymbol{h}_{m, i}=\left[T_{m, 1}\left(z_{i}\right) T_{m, 2}\left(z_{i}\right)\right], i=1,2, \ldots, N$

Using matrix notation, Eq. (15) becomes the following linear form:

$$
\boldsymbol{A}_{m} \boldsymbol{x}_{m} \leq \boldsymbol{b}_{m}
$$

where matrix $\boldsymbol{A}_{m}$ and vector $\boldsymbol{b}_{m}$ are given by:

$$
\boldsymbol{A}_{m}=\left[\begin{array}{c}
\boldsymbol{A}_{m, 1} \\
\boldsymbol{A}_{m, 2} \\
\vdots \\
\boldsymbol{A}_{m, N}
\end{array}\right], \boldsymbol{b}_{m}=\left[\begin{array}{c}
\boldsymbol{b}_{m, 1} \\
\boldsymbol{b}_{m, 2} \\
\vdots \\
\boldsymbol{b}_{m, N}
\end{array}\right] .
$$

At the end, the design problem can be cast to the typical optimization one:

$\min _{\boldsymbol{x}_{m}} \frac{1}{2}\left\|\boldsymbol{c}_{m} \boldsymbol{x}_{m}-d_{m}\right\|_{2}^{2}$ subject to:

$\boldsymbol{A}_{m} \boldsymbol{x}_{m} \leq \boldsymbol{b}_{m}$

It should be mentioned that the linear programming (LP) technique can also be used.

\section{DESIGN EXAMPLE}

This section shows two design cases. The first case considers only limitation of the gain in the passband, in the absence of constrains in the stopbands. The value of the maximum gain in the passband is settled to 1.005 , $\left(\delta_{m}^{P B}=1.005\right)$. In the second case, a stringent condition in the stopband is included, while constrain in the passband is cancelled. An attenuation of $30 \mathrm{~dB}\left(\delta_{m}^{S B}=0.032\right)$ is requested. It should be noted that the maximum attenuation of $T_{m}^{R}(z)$ cannot be higher than the attenuation of $T_{m, 0}(z)$, that is about $40 \mathrm{~dB}$. The fundamental frequency $f_{1}=50 \mathrm{~Hz}$ and the sampling frequency of $f_{\mathrm{s}}=800 \mathrm{~Hz}$ have been adopted.

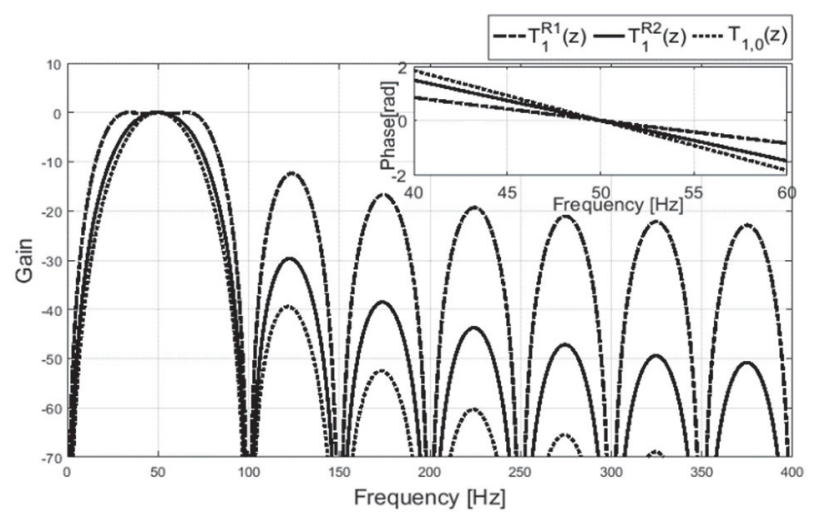

Figure 5 Frequency responses of the transfer functions $T_{m}^{R 1}(z), T_{m}^{R 2}(z)$, and $T_{m, 0}(z)$, corresponding to the fundamental component $(m=1)$, with $f_{1}=50 \mathrm{~Hz}$ and $f_{\mathrm{s}}=800 \mathrm{~Hz}$

Fig. 5 shows the amplitude and phase frequency responses of the fundamental component of $T_{m, 0}(z)(m=1)$, together with those of $T_{m}^{R 1}(z)$ (for $r_{m, 1}=0.5293$ and $r_{m, 2}=$ 0.2490 ) and $T_{m}^{R 2}(z)$ (for $r_{m, 1}=0.2013$ and $\left.r_{m, 2}=0.0004\right)$. Note that although both $T_{m}^{R 1}(z)$ and $T_{m}^{R 2}(z)$ provide flattop-amplitude responses around the actual harmonic frequency, $T_{m}^{R 1}(z)$ provides a wider passband on one hand, 
and higher side lobes on the other one. The high interharmonic gains are considerably mitigated in comparison with $T_{m}^{F}(z)$, and the zero-flat gains in the stopbands are preserved. However, the phase response in the passband is no longer zero-flat $\quad\left(\left|\mathrm{d} T_{m}^{R 1}(z) / d z\right|_{z=z_{m}}=11.0603 \quad\right.$ and $\left.\left|\mathrm{d} T_{m}^{R 2}(z) / d z\right|_{z=z_{m}}=18.7695\right)$. However, these group delays are smaller than for $T_{m, 0}\left(\left|\mathrm{~d} T_{m, 0}(z) / d z\right|_{z=z_{m}}=23.50\right)$, allowing a smaller latency. It is also visible from the phase time responses that the group delay for $T_{m}^{R 1}(z)$ is smaller than for $T_{m}^{R 2}(z)$. On the other hand, side lobes are much larger for $T_{m}^{R 1}(z)$ than for $T_{m}^{R 2}(z)$, which decreases a robustness against interharmonics and noise. It is possible to observe that the $T_{m}^{R 1}(z)$ filter yields an excessive bandwidth at the expense of a weaker stopband attenuation, which is detrimental to the estimator interference rejection capability. In the next section, simulations with two performance tests will be performed to analyse the influence of the chosen constrains, and consequently designed parameters, on tracking performance.

Due to space savings, only frequency responses for the basic component $(m=1)$ are depicted. The frequency responses for other harmonics have the same shapes. It is important to note that, for asynchronous sampling, the parameters designed for one harmonic also apply to the others.

\section{SIMULATION RESULTS}

The aim of the following examples is only to demonstrate the ability of the algorithm to track the fundamental and harmonic components, but not to demonstrate a compliance with any standard. Tests have been performed by computer simulations. A sampling frequency of $f_{\mathrm{s}}=800 \mathrm{~Hz}$ was adopted. A multiple harmonic content is injected to the signal. Odd harmonics (up to seventh) were added simultaneously to the signal waveform, with the individual harmonic levels $30 \%, 10 \%$ and $10 \%$, respectively. Even harmonics are set to zero. Parameters (coefficients $r_{m, 1}$ and $r_{m, 2}$ corresponding group delay) of all considered cases are summarized in Tab. 1.

The estimates of the amplitude, phase, and TVE in percentage, obtained by $V_{m}^{F}(z), V_{m}^{R 1}(z), V_{m}^{R 2}(z)$, and $V_{m, 0}$, with $f_{1}=50 \mathrm{~Hz}$ and $f_{\mathrm{s}}=800 \mathrm{~Hz}$, for the phase angle modulated and step changes, are shown in Fig. 6 and Fig. 7.

Table 1 Parameters of considered cases

\begin{tabular}{|c|c|c|c|}
\hline Transfer function & $r_{m, 1}$ & $r_{m, 2}$ & Group delay \\
\hline$T_{m, 0}(z)$ & 0 & 0 & 23.5000 \\
\hline$T_{m}^{1 D}(z)$ & 1 & 0 & 0 \\
\hline$T_{m}^{F}(z)$ & 1 & 1 & 0 \\
\hline$T_{m}^{R 1}(z)$ & 0.5293 & 0.2490 & 11.0603 \\
\hline$T_{m}^{R 2}(z)$ & 0.2013 & 0.0004 & 18.7695 \\
\hline
\end{tabular}

Similar performances were obtained for changes of the phasor amplitude too. Given the brevity, estimates for the basic component are shown only. The waveforms of estimates for harmonic phasors have similar shapes.

A total vector error (TVE) is defined by Eq. (18), and it is used as an index of cumulative information, which aggregates three possible sources of error: amplitude, phase angle, and timing [2].

$$
T V E(n)=\sqrt{\frac{\left[\hat{X}_{r}(n)-X_{r}(n)\right]^{2}+\left[\hat{X}_{i}(n)-X_{i}(n)\right]^{2}}{\left[X_{r}(n)\right]^{2}+\left[X_{i}(n)\right]^{2}}}
$$

where $\hat{X}_{r}(n)$ and $\hat{X}_{i}(n)$ are the sequences of estimates given by the measuring device, $\hat{X}(n)=\hat{X}_{r}(n)+j \hat{X}_{i}(n)$, and $X_{r}(n), X_{i}(n), X(n)=X_{r}(n)+j X_{i}(n)$, are the corresponding theoretical values of the input signal at the instant of measurement [35]. The TVE is thus a convenient mean to express the accuracy of measurement. This representation offers the advantage of being extremely compact, but it is often more useful to refer back to individual errors components, to understand better the impact of measurement accuracy on the specific application [2].

The first test is referred to the phase modulation of the input signal, reflecting phase angle variations that can occur in the voltage waveforms during a swing of the stable power. The standard [35] proposes the following test signal:

$s(t)=X_{m} \cos \left[2 \pi f_{1} t+k_{a} \cos \left(2 \pi f_{m} t-\pi\right)\right]$

where $X_{m}$ is an amplitude of the fundamental component, $k_{a}$ and $f_{m}$ are an amplitude and a frequency of the phase modulation signal, respectively. IEC/IEEE Standard 60255-118-1:2018 [35] proposes the separate amplitude and phase modulation tests. In this paper an example with a phase angle modulation is presented. The following parameters are considered: $X_{m}=1(p . u),. k_{a}=0.1 \mathrm{rad}$ and $f_{m}=$ $2 \mathrm{~Hz}$.

From Fig. 6, a large systematic delay is visible for the estimates obtained by $V_{m, 0}$, where the maximum value of TVE of about $3.8 \%$ is obtained. For the estimates obtained by $V_{m}^{R 1}(z)$ and $V_{m}^{R 2}(z)$ the maximum values of TVE are smaller. The maximum TVEs obtained utilizing $V_{m}^{R 1}(z)$ and $V_{m}^{R 2}(z)$ are about $1.9 \%$ and $3 \%$, respectively. In the $V_{m}^{F}(z)$ case, the maximum value of TVE is very small. The group delay for $V_{m}^{R 1}(z)$ case is a half of one obtained by $V_{m, 0}$. For $V_{m}^{R 2}(z)$ case the group delay and TVE are lager in comparison with $V_{m}^{R 1}(z)$ case due to narrower passband, while attenuation in the stopband is greater providing a better immunity to the interharmonic and noise interference. In this test, the obtained performance by utilizing $V_{m}^{R}(z)$ is better than for $V_{m, 0}$ case, and worse than in $V_{m}^{F}(z)$ one. 
The test signal referred to the phase angle step change has the following form

$$
s(t)=X_{m} \cos \left[2 \pi f_{1} t+k_{a} u\left(t-t_{S}\right)\right]
$$

where $X_{m}$ is an amplitude of the fundamental component, $u(t)$ is a step function with a unity amplitude and $t_{s}$ is a time instant of the step occurrence. The phase step with $k_{a}=\pi / 18$ $\left(10^{\circ}\right.$ of the step of the phase angle) is applied to the signal in the instant $t_{s}=0 \mathrm{~s}$. The duration of the transition times is three cycles of the fundamental component. The estimates obtained by $V_{m}^{F}(z)$ have a huge overshoot, that is a consequence of the large gain peaks at the resonant frequencies in the frequency response (Fig. 3). Estimate obtained by usage of $V_{m}^{R 1}(z)$ shows a significantly lower overshoot, equal to about $10 \%$, and by $V_{m}^{R 2}(z)$ without overshoot, Fig. 7. In case $V_{m}^{R 2}(z)$, TVE falls to near zero within the first two cycles, while for $V_{m}^{R 1}(z)$ decreases very fast below $3 \%$ within the first cycle. Like in the previous test, $T_{m}^{R 1}(z)$ and $T_{m}^{R 2}(z)$ exhibit performances that lay between two extreme cases, but now better than in $T_{m}^{F}(z)$ and worse than in $T_{m, 0}$ case. It can be concluded that performances of $T_{m}^{R 1}(z)$ need to be improved in the step test, but they are better in terms of reducing the group delay and improving tracking of the modulated signals.

It is obvious that a wider passband provides a lower latency, but resulted overshoots for inputs with step changes are much larger. And vice versa, a reduction in bandwidth provides lower overshoots for step-changed input responses and longer response times and group delays. The bandwidth modification allows a trade-off between these opposite requirements.

\section{CONCLUSION}

This paper studies the spectral estimation based on the MR filters presented in [31]. In this paper, the CLLS optimization technique is applied to find a compromised solution between the conflicting requirements arising from the need for instantaneous estimation, which is achieved by sufficient both amplitude and phase frequency responses flatness in the bandwidth centre, and admissible levels of peaks at the bandwidth ends. The results of the conducted computer simulations showed that the obtained performances of this estimation algorithm are in the middle compared to the best and the worst case obtained for two extreme inherent cases. Thus, the obtained estimators can be at the same time sufficiently accurate and resistant to the harmonics and/or interharmonics and the presence of noise, and yet fast enough to enable monitoring of the content of nonstationary signals.
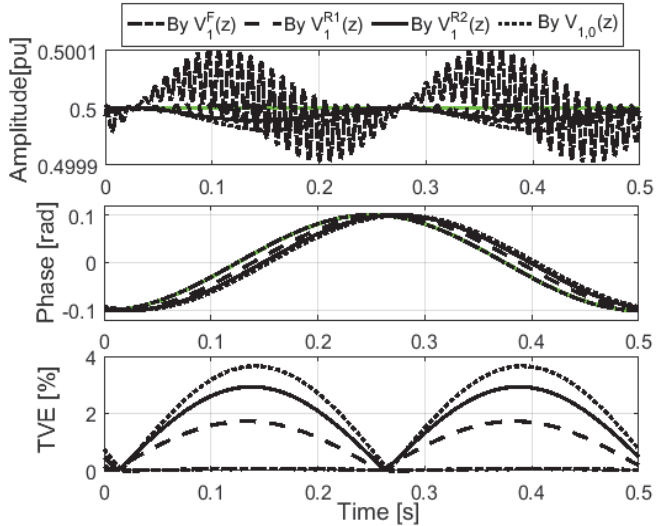

Figure 6 Estimation in case of the phase angle modulated signal, for $V_{m}^{F}(z)$, $V_{m}^{R 1}(z), V_{m}^{R 2}(z)$ and $V_{m, 0}$, for the fundamental component $(m=1)$, with $f_{1}=$ $50 \mathrm{~Hz}$ and $f_{\mathrm{s}}=800 \mathrm{~Hz}$
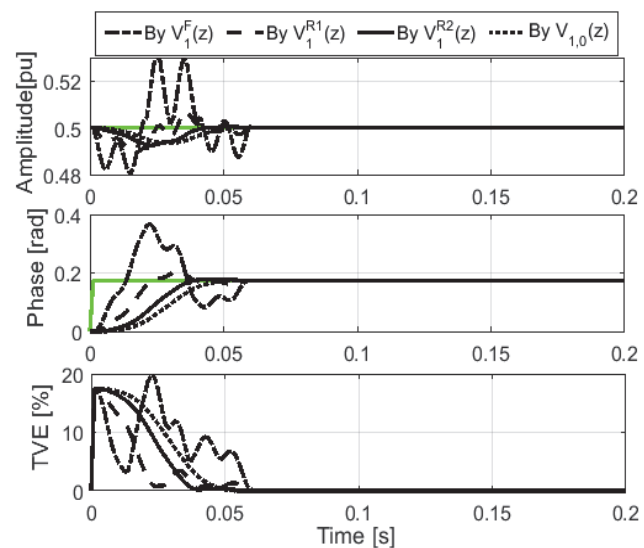

Figure 7 Estimation case of the phase angle step changed signal, for $V_{m}^{F}(z)$, $V_{m}^{R 1}(z), V_{m}^{R 2}(z)$ and $V_{m, 0}$, for the fundamental component $(m=1)$, with $f_{1}=$ $50 \mathrm{~Hz}$ and $f_{\mathrm{s}}=800 \mathrm{~Hz}$

It is worth noting the possibility of obtaining a set of filters based on the core MR-structure, to simultaneously estimate different harmonic phasor with different estimation performances. For example, the fundamental component and dominant low order harmonics can be estimated with a smaller group delay and faster response, since latency may be more important than accuracy for feedback control in many applications such as wide-area control and protection, while low level harmonic phasors can be estimated with filters with a narrow bandwidth and a slow response. Even more, the same harmonic phasor can be estimated with different dynamics and so obtained estimates further be used for different purposes.

Further works will be focused to the compliance of this estimation technique with the relevant standards.

\section{REFERENCES}

[1] Barros, J., Diego, R. I., \& de Apraiz, M. (2013). A discussion of new requirements for measurement of harmonic distortion in modern power supply systems. IEEE Transactions on Instrumentation and Measurement, 62(8), 2129-2139. https://doi.org/10.1109/TIM.2013.2267451

[2] Castello, P., Lixia, M., Muscas, C., \& Pegoraro, P. A. (2012). Impact of the model on the accuracy of synchrophasor measurement. IEEE Transactions on Instrumentation and Measurement, 61(8), 2179-2188. https://doi.org/10.1109/TIM.2012.2193699 
[3] Phadke, A. G. \& Kasztenny, B. (2008). Synchronized phasor and frequency measurement under transient conditions. IEEE transactions on power delivery, 24(1), 89-95. https://doi.org/10.1109/TPWRD.2008.2002665

[4] Mai, R. K., He, Z. Y., Fu, L., Kirby, B., \& Bo, Z. Q. (2010). A dynamic synchrophasor estimation algorithm for online application. IEEE Transactions on Power Delivery, 25(2), 570-578. https://doi.org/10.1109/TPWRD.2009.2034293

[5] Barchi, G., Macii, D., \& Petri, D. (2013). Synchrophasor estimators accuracy: A comparative analysis. IEEE Transactions on Instrumentation and Measurement, 62(5), 963-973. https://doi.org/10.1109/TIM.2012.2236776

[6] Belega, D., Macii, D., \& Petri, D. (2013). Fast synchrophasor estimation by means of frequency-domain and time-domain algorithms. IEEE Transactions on Instrumentation and Measurement, 63(2), 388-401. https://doi.org/10.1109/TIM.2013.2279000

[7] de Carvalho, J. R., Duque, C. A., Lima, M. A., Coury, D. V., \& Ribeiro, P. F. (2014). A novel DFT-based method for spectral analysis under time-varying frequency conditions. Electric Power Systems Research, 108, 74-81. https://doi.org/10.1016/j.epsr.2013.10.017

[8] Marques, C. A., Ribeiro, M. V., Duque, C. A., Ribeiro, P. F., $\&$ da Silva, E. A. (2011). A controlled filtering method for estimating harmonics of off-nominal frequencies. IEEE Transactions on Smart Grid, 3(1), 38-49. https://doi.org/10.1109/TSG.2011.2174259

[9] Girgis, A. A., Chang, W. B., \& Makram, E. B. (1991). A digital recursive measurement scheme for online tracking of power system harmonics. IEEE Transactions on Power Delivery, 6(3), 1153-1160. https://doi.org/10.1109/61.85861

[10] Saiz, V. M. \& Guadalupe, J. B. (1997). Application of Kalman filtering for continuous real-time tracking of power system harmonics. IEE Proceedings-Generation, Transmission and Distribution, 144(1), 13-20. https://doi.org/10.1049/ip-gtd:19970550

[11] Chen, C. I., Chang, G. W., Hong, R. C., \& Li, H. M. (2009). Extended real model of Kalman filter for time-varying harmonics estimation. IEEE Transactions on Power Delivery, 25(1), 17-26. https://doi.org/10.1109/TPWRD.2009.2035217

[12] Mojiri, M., Karimi-Ghartemani, M., \& Bakhshai, A. (2010). Processing of harmonics and interharmonics using an adaptive notch filter. IEEE transactions on power delivery, 25(2), 534-542. https://doi.org/10.1109/TPWRD.2009.2036624

[13] Terzija, V. \& Stanojevic, V. (2007). Two-stage improved recursive Newton-type algorithm for power-quality indices estimation. IEEE Transactions on Power delivery, 22(3), 1351-1359. https://doi.org/10.1109/TPWRD.2007.899565

[14] Singh, S. K., Sinha, N., Goswami, A. K., \& Sinha, N. (2016). Robust estimation of power system harmonics using a hybrid firefly based recursive least square algorithm. International Journal of Electrical Power \& Energy Systems, 80, 287-296. https://doi.org/10.1016/j.jijepes.2016.01.046

[15] Sadinezhad, I. \& Agelidis, V. G. (2012). Real-time power system phasors and harmonics estimation using a new decoupled recursive-least-squares technique for DSP implementation. IEEE Transactions on industrial electronics, 60(6), 2295-2308. https://doi.org/10.1109/TIE.2012.2192895

[16] Ray, P. K., Puhan, P. S., \& Panda, G. (2016). Real time harmonics estimation of distorted power system signal. International Journal of Electrical Power \& Energy Systems, 75, 91-98. https://doi.org/10.1016/j.ijepes.2015.08.017

[17] Karimi-Ghartemani, M. \& Iravani, M. R. (2002). A nonlinear adaptive filter for online signal analysis in power systems: Applications. IEEE Transactions on Power Delivery, 17(2), 617-622. https://doi.org/10.1109/61.997949

[18] Karimi-Ghartemani, M. \& Iravani, M. R. (2005). Measurement of harmonics/inter-harmonics of time-varying frequencies. IEEE Transactions on Power Delivery, 20(1), 23-31. https://doi.org/10.1109/TPWRD.2004.837674

[19] McNamara, D. M., Ziarani, A. K., \& Ortmeyer, T. H. (2006). A new technique of measurement of nonstationary harmonics. IEEE transactions on power delivery, 22(1), 387395. https://doi.org/10.1109/TPWRD.2006.874622

[20] de Carvalho, J. R., Duque, C. A., Ribeiro, M. V., Cerqueira, A. S., Baldwin, T. L., \& Ribeiro, P. F. (2009). A PLL-based multirate structure for time-varying power systems harmonic/interharmonic estimation. IEEE Transactions on Power Delivery, 24(4), 1789-1800. https://doi.org/10.1109/TPWRD.2009.2027474

[21] Barros, J., Diego, R. I., \& De Apraiz, M. (2012). Applications of wavelet transform for analysis of harmonic distortion in power systems: A review. IEEE Transactions on instrumentation and measurement, 61(10), 2604-2611. https://doi.org/10.1109/TIM.2012.2199194

[22] Yang, J. Z., Yu, C. S., \& Liu, C. W. (2005). A new method for power signal harmonic analysis. IEEE Transactions on Power Delivery, 20(2), 1235-1239. https://doi.org/10.1109/TPWRD.2004.834311

[23] Sohn, S. W., Lim, Y. B., Yun, J. J., Choi, H., \& Bae, H. D. (2011). A filter bank and a self-tuning adaptive filter for the harmonic and interharmonic estimation in power signals. IEEE Transactions on Instrumentation and Measurement, 61(1), 64-73. https://doi.org/10.1109/TIM.2011.2150610

[24] Kušljević, M. D. (2008). Adaptive resonator-based method for power system harmonic analysis. IET science, measurement \& technology, 2(3), 177-185. https://doi.org/10.1049/iet-smt:20070068

[25] Duque, C. A., Silveira, P. M., \& Ribeiro, P. F. (2011). Visualizing time-varying harmonics using filter banks. Electric power systems research, 81(4), 974-983. https://doi.org/10.1016/j.epsr.2010.11.030

[26] Platas-Garza, M. A. \& de la O Serna, J. A. (2010). Dynamic harmonic analysis through Taylor-Fourier transform. IEEE Transactions on Instrumentation and Measurement, 60(3), 804-813. https://doi.org/10.1109/TIM.2010.2064690

[27] De la O Serna, J. A. \& Rodríguez-Maldonado, J. (2012). Taylor-Kalman-Fourier filters for instantaneous oscillating phasor and harmonic estimates. IEEE Transactions on Instrumentation and Measurement, 61(4), 941-951. https://doi.org/10.1109/TIM.2011.2178677

[28] Liu, J., Ni, F., Pegoraro, P. A., Ponci, F., Monti, A., \& Muscas, C. (2012, September). Fundamental and harmonic synchrophasors estimation using modified Taylor-Kalman filter. 2012 IEEE International Workshop on Applied Measurements for Power Systems (AMPS) Proceedings, 1-6. https://doi.org/10.1109/AMPS.2012.6344004

[29] Roscoe, A. J., Abdulhadi, I. F., \& Burt, G. M. (2013). P and $M$ class phasor measurement unit algorithms using adaptive cascaded filters. IEEE transactions on power delivery, 28(3), 1447-1459. https://doi.org/10.1109/TPWRD.2013.2238256

[30] Chen, C. I. \& Chen, Y. C. (2013). Comparative study of harmonic and interharmonic estimation methods for stationary and time-varying signals. IEEE Transactions on Industrial Electronics, 61(1), 397-404. https://doi.org/10.1109/TIE.2013.2242419

[31] Kušljević, M. D. \& Tomić, J. J. (2014). Multiple-resonatorbased power system Taylor-Fourier harmonic analysis. IEEE Transactions on Instrumentation and Measurement, 64(2), 554-563. https://doi.org/10.1109/TIM.2014.2345591

[32] Kušljević, M. D., Tomić, J. J., \& Poljak, P. D. (2017). Maximally flat-frequency-response multiple-resonatorbased harmonic analysis. IEEE Transactions on Instrumentation and Measurement, 66(12), 3387-3398. https://doi.org/10.1109/TIM.2017.2751799

[33] Tseng, C. C. \& Lee, S. L. (2002). Minimax design of stable IIR digital filter with prescribed magnitude and phase responses. IEEE Transactions on Circuits and Systems I: 
Fundamental Theory and Applications, 49(4). https://doi.org/10.1109/81.995676

[34] Gill, P. E., Murray, W., \& Wright, M. H. (2019). Practical optimization. Society for Industrial and Applied Mathematics. https://doi.org/10.1137/1.9781611975604

[35] (2018). IEC/IEEE 60255-118-1:2018: IEEE/IEC International Standard - Measuring relays and protection equipment Part 118-1: Synchrophasor for power systems - Measurements. IEEE.

\section{Contact information:}

Miodrag D. KUŠLJEVIĆ, Dr. executive manager

(Corresponding author)

Termoelektro Enel AD,

11010 Belgrade, Bačvanska 21/III, Serbia

E-mail: Miodrag.Kusljevic@te-enel.rs

Josif J. TOMIĆ, Dr. associate professor

University of Novi Sad,

21000 Novi Sad, Trg Dositeja Obradovića 6, Serbia

E-mail: tomicj@uns.ac.rs

Predrag D. POLJAK, Dr. research associate

Institute of Chemistry, Technology and Metallurgy,

11000 Beograd, Njegoševa 12, Serbia

E-mail: Predrag.Poljak@nanosys.ihtm.bg.ac.rs 